\title{
Enhanced Quantum Efficiency in Mixed Donor-Acceptor Nanocrystal Quantum Dot Monolayers
}

\author{
Xia Zhang ${ }^{1}$, Manuela Lunz' ${ }^{1}$, Valerie A. Gerard ${ }^{2}$, Yurii K. Gun'ko², Vladimir Lesnyak ${ }^{3}$, Nikolai Gaponik ${ }^{3}$ \\ and A. Louise Bradley ${ }^{*}$, \\ ${ }^{1}$ Semiconductor Photonics Group, School of Physics, Trinity College Dublin, Dublin 2, Ireland \\ ${ }^{2}$ School of Chemistry, Trinity College Dublin, Dublin 2, Ireland \\ ${ }^{3}$ Physical Chemistry, TU Dresden, Bergstr. 66b, 01062 Dresden, Germany \\ *Tel: +353 (0)1 896 3595, Fax: +353 (0)1 67 11759, e-mail: bradlel@tcd.ie
}

\begin{abstract}
Colloidal nanocrystal quantum dots have attracted interest for use in light-harvesting and emitting devices. One of the key parameters for such applications is the quantum efficiency. Nanocrystal quantum dots exhibit reduced quantum yield in layers or solids compared with solutions. Optimizing the overall efficiency of the emission is an important goal. Here we report on a large enhancement of the quantum efficiency of mixed TGA-stabilised CdTe quantum dot in closely packed monolayers formed with nanocrystal QDs of two sizes. Energy is transferred from the smaller donor quantum dots to the larger acceptor quantum dots via Förster resonant energy transfer (FRET). The increased quantum efficiency is attributed to exciton recycling from the donor defect states. The acceptor emission enhancement and relative quantum efficiency are strongly dependent on the donor:acceptor concentration ratio. A relative quantum efficiency of $160 \%$ is achieved for the mixed quantum dot layer with a donor:acceptor ratio of 11:1.
\end{abstract}

Keywords: Förster resonant energy transfer, nanocrystal quantum dots, quantum efficiency

\section{INTRODUCTION}

Semiconductor nanocrystal quantum dots (QDs) are extensively researched for applications in solid state lighting, photovoltaics and sensing [1]. Their many advantages include size tunable emission, narrow emission linewidth, broad absorption and good photostability [2]. In addition their colloidal nature makes them suitable for a range of relatively simple large area deposition techniques such as spin coating and dip-coating. A number of nanocrystal device designs are based on the principle of Förster resonant energy transfer (FRET), as a means of pumping or extracting energy from the QDs. FRET is an energy transfer mechanism from a donor to an acceptor species by dipole-dipole interactions [3]. FRET has also been proposed for colour tuning [4] and white light generation in nanocrystal light emitting devices [5]. It has been observed that despite having a relatively high quantum yields in solution, the nanocrystal QDs exhibit a much reduced QY in films which would limit the overall quantum efficiency [6]. Reports by Franzl et al. [7] and more recently by Nizamoglu et al. [8] suggest that energy transfer from the donor to the acceptor QDs occurs not only from the bright donor states but can also occur from the "dark" donor defect states. Therefore, FRET can provide a mechanism to recycle the excitons trapped in the donor defect states and increase the overall quantum efficiency of the QD-based light emitted. Franzl et al. reported reported a four-fold increase the acceptor emission in a structure comprised of 7 layers of four different sizes of QDs. The size graded structure funnelled energy from the smaller QDs to the larger QDs, achieving a 28-fold increase in the exciton concentration in the final acceptor layer [7]. Nizamoglu et al. report a $17 \%$ enhancement of the quantum efficiency in a mixed solid comprised of two different sizes of QD for a 1:1 donor:acceptor ratio [8].

In this paper we report on the concentration dependence of the acceptor emission and the emission efficiency in closely packed mixed CdTe QD monolayers. As will be seen the enhancement of the acceptor emission due to FRET from the acceptor QDs is greater than expected from the donor quenching and at a donor:acceptor concentration of $11: 1$ a relative emission efficiency of $160 \%$ is achieved.

\section{Experimental Methods}

Two different sizes of negatively charged CdTe nanocrystal QDs, stabilized by thioglycolic acid in aqueous solution [9], were deposited in pure and mixed monolayers on quartz slides. The $2.9 \mathrm{~nm}$ and $3.5 \mathrm{~nm}$ diameter QDs acted as the energy donors and energy acceptors, respectively. Closely packed monolayers are deposited using the layer-by-layer (LbL) electrostatic assembly technique [10]. Monolayers with either one or two types of QD dots, with varying concentration ratios, can be prepared by immersion in pure or mixed QD solutions. Further details on the sample preparation can be found in reference [11]. As can be seen in Fig. 1(a), in the pure monolayers the donors QDs emission is centred at $550 \mathrm{~nm}$ and the acceptor emission is centred at $616 \mathrm{~nm}$.

A double beam UV-Vis Recording Spectrometer (Shimadzu UV-2401 PC) was used to record the absorption spectra of the QD structures. The room temperature steady-state photoluminescence (PL) spectra were measured with a Perkin-Elmer LS 55 fluorescence spectrometer with an excitation wavelength of $400 \mathrm{~nm}$. Time-resolved 
PL decays were recorded with a PicoQuant Microtime200 time-resolved confocal microscope system with 150 ps resolution. A LDH-480 laser head controlled by a PDL-800B driver (PicoQuant) provided picosecond pulses at $470 \mathrm{~nm}$ for excitation. Areas of 80 by $80 \mu \mathrm{m}$ (150 by 150 pixels) were scanned during the measurements, with integration times of $4 \mathrm{~ms}$ per pixel and a repetition rate of $10 \mathrm{MHz}$. A broad band filter (FWHM: $70 \pm 5 \mathrm{~nm}$ ) centred at $500 \mathrm{~nm}$ was used to isolate the donor PL decay in the measurements of the pure donor and mixed QD monolayers reference.

\section{Results and Discussion}

Firstly the spectral properties of the reference and mixed QD monolayers will be presented. After which the concentration dependence of the donor quenching, acceptor emission enhancement and quantum efficiency will be discussed.

\subsection{FRET in a mixed QD monolayer with a 1:1 donor:acceptor concentration ratio}

The PL spectra for pure reference donor and acceptor monolayers, as well as a mixed monolayer with the same donor and acceptor QD concentrations of $(1.0 \pm 0.2) \times 10^{17} \mathrm{~m}^{-2}$, corresponding to a 1:1 donor:acceptor ratio, are shown in Fig. 1(a). A quantum yield of 5\% and 7\% was estimated for the pure donor and acceptor monolayers, respectively. The QD size and concentration in the pure monolayers can be extracted from the position of the first absorption peak and the absolute value at this wavelength [12]. The concentrations of the QDs in the mixed monolayers were determined by fitting the mixed film absorption spectrum by a weighted sum of the pure acceptor and donor monolayer absorption spectra. By comparison with the reference monolayers, it can be seen that in the mixed monolayer the donor emission is quenched and the acceptor emission is enhanced.
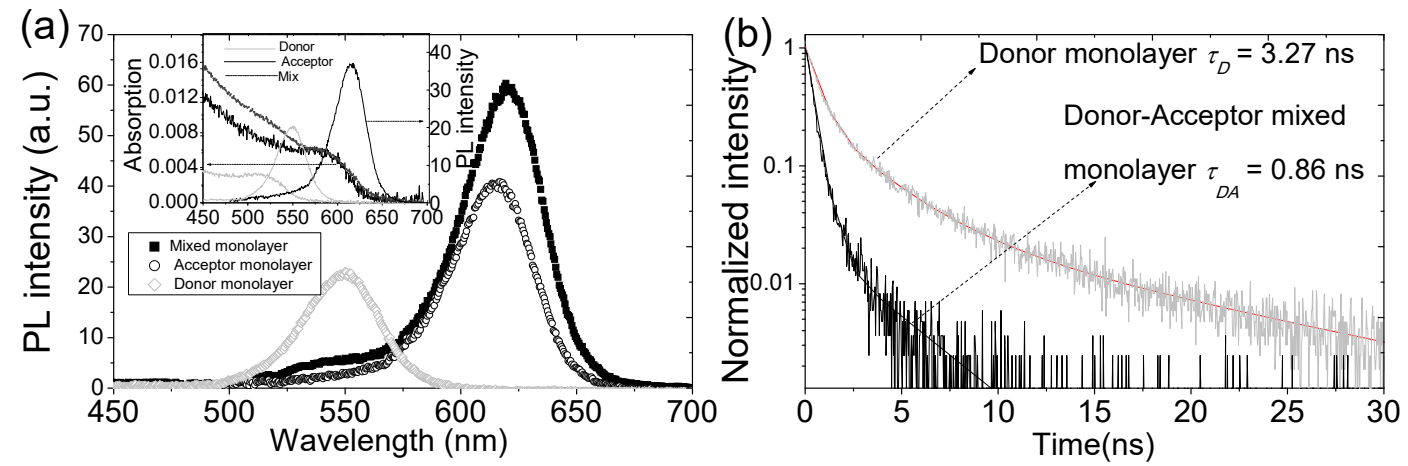

Figure 1. (a) Photoluminescence (PL) spectrum of a mixed donor-acceptor monolayer (black squares) with a equal donor and acceptor concentrations of $(1.0 \pm 0.2) \times 10^{17} \mathrm{~m}^{-2}$. The PL spectra of the donor and acceptor

reference monolayers with the same donor and acceptor concentrations are also shown. In the inset the absorption and PL spectra of the references monolayers, as well as the absorption of the mixed monolayer, are shown. (b) Donor PL decays of the donor-acceptor mixed monolayer (black line) and the donor reference monolayer (grey line).

The donor emission quenching, $Q$, which is also a measure of the FRET efficiency, and the acceptor enhancement, $E_{E N H}$, are defined as

$$
Q=1-\frac{I_{D A}}{I_{D}} \text { and } E_{E N H}=\frac{I_{A D}}{I_{A}}-1
$$

where $I_{D}$ and $I_{A}$ are the integrated emission for the pure donor and acceptor reference monolayers, respectively, and $I_{D A}$ and $I_{A D}$ are the integrated donor and acceptor emission in the mixed monolayer, respectively. In the example shown in Fig. 1(a), the observed donor quenching, $Q$, of $60 \%$ and the acceptor enhancement of 0.35 are typical signatures of FRET. Further evidence for FRET is seen in the decrease of the donor PL lifetime in the presence of the acceptors in the mixed monolayer, as can be seen in Fig. 1(b). The average donor lifetime, calculated using a two-exponential fit for both the pure donor and mixed QD monolayers, decreases from $\tau_{D}=(3.3 \pm 0.2) \mathrm{ns}$ in the pure donor monolayer to $\tau_{D A}=(0.9 \pm 0.2) \mathrm{ns}$ in the mixed monolayer, corresponding to a FRET efficiency of $E_{F R E T}=1-\frac{\tau_{D A}}{\tau_{D}}$ of $\sim 74 \%$ at this donor:acceptor ratio. This is greater than the FRET efficiency measured from the donor PL quenching, $Q$, due to the influence of 
intra-ensemble energy transfer which measured on the blue side of the donor emission spectrum which influences the FRET efficiency measured from the lifetime data $[13,14]$. For the rest of the discussion on the emission properties only the FRET efficiency evaluated from the PL spectra will be considered.
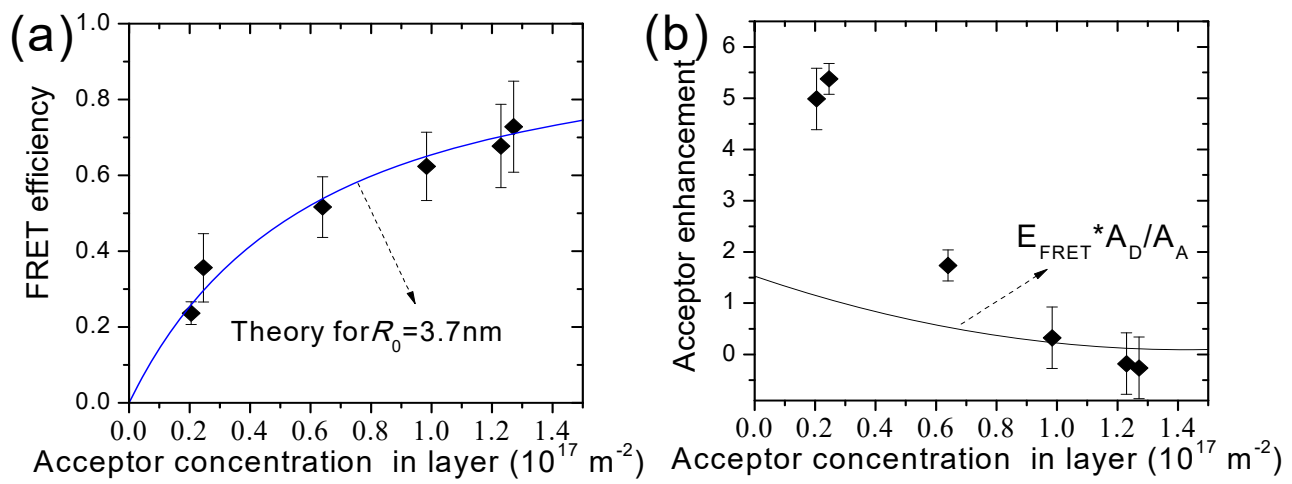

Figure 2. (a) Acceptor concentration dependence of the FRET efficiency evaluated from the donor $P L$ quenching, $Q$. The solid line shows the agreement with theory with the Förster radius, $R_{0}=3.7 \mathrm{~nm}$, which

agrees within the error with the Förster radius calculated from the spectral overlap [11]. (b) Acceptor concentration dependence of the acceptor enhancement. The acceptor enhancement expected from theory is shown by the solid line.

\subsection{Concentration Dependence}

To further investigate the emission properties of the mixed monolayer, samples with varying donor:acceptor ratio were prepared. Firstly, the concentration dependence of the FRET efficiency is shown in Fig. 2 (a). The measured concentration dependence of the FRET efficiency is in good agreement with the theory [11]. As expected the FRET efficiency decreases as the acceptor concentration decreases, corresponding to an increasing donor:acceptor ratio in the closely packed mixed monolayer. A FRET efficiency of $70 \%$ is observed at an acceptor concentration of $(1.3 \pm 0.1) \times 10^{17} \mathrm{~m}^{-2}$ corresponding to a donor:acceptor ratio of 0.5 . The acceptor concentration dependence of the acceptor enhancement can be seen in Fig. 2(b). It shows the opposite dependence to the FRET efficiency, with a maximum value of $\sim 5.5$ at an acceptor concentration of $(0.2 \pm 0.1) \times 10^{17} \mathrm{~m}^{-2}$, corresponding to a donor:acceptor ratio of 11:1. The acceptor enhancement arising from FRET can be calculated by multiplying the FRET efficiency by the ratio of the donor:acceptor absorption ratio, $E_{E N H}=\frac{A_{D}}{A_{A}} E_{F R E T}$ [15]. The calculated acceptor enhancement, also shown in Fig. 2(b), clearly underestimates the experimentally measured acceptor enhancement. The difference is attributed to recycling of excitons from the "dark" donor defect states to the acceptor. This is not considered in the calculated acceptor enhancement based on the FRET efficiency evaluated from the quenching of the bright donor states.

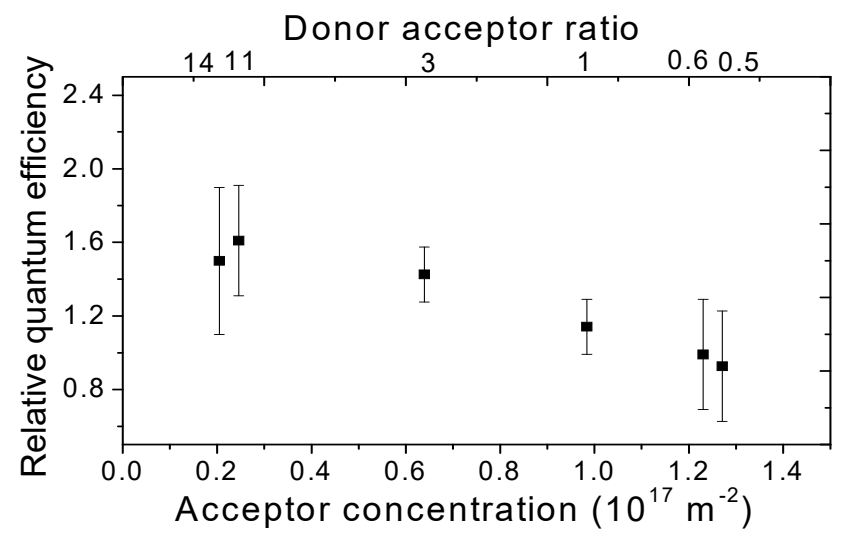

Figure 3. Dependence of the relative quantum efficiency of the mixed donor-acceptor monolayer on the acceptor concentration. The donor:acceptor ratio in the mixed monolayer is shown on the top axis. 
The recycling effect can also have a large effect on the overall emission of the mixed monolayer. The relative quantum yield is defined as

$$
Q E=\frac{I_{\operatorname{mix}}}{I_{D}+I_{A}}
$$

where $I_{D}$ and $I_{A}$ are the integrated emission from the pure monolayers and $I_{m i x}$ is the integrated emission from the mixed monolayer with similar donor and acceptor concentrations. As can be seen in Fig. 3 the relative quantum yield is strongly dependent on the donor:acceptor ratio, with a value of $\sim 1.6$ at a ratio of 11:1. The acceptor QDs have a slightly higher quantum yield than the donor QDs which would be expected to result in a relative quantum yield greater than 1 , as excitons transferred from the donors to the acceptors have a higher probability of radiative recombination. However the small increase in quantum yield cannot account for the large increase in the mixed monolayer emission, which is again attributed to exciton recycling from the donor defect states. It can also be noted that no recycling is observed in pure donor or acceptor monolayers indicating that the spectral overlap between the donors and acceptors is an important factor in the recycling efficiency.

\section{Conclusion}

In summary, the concentration dependence the emission properties of mixed CdTe QD monolayers has been investigated. Energy is transferred from donor to acceptor QDs via FRET. A large acceptor enhancement is observed for the mixed monolayers. The acceptor emission in the mixed monolayer with a donor:acceptor ratio of 11:1 is increased by more than 5 times the emission of a pure acceptor monolayer of the same QD concentration. The experimentally measured enhancement is more than four times greater than that expected from the theory and is attributed to exciton recycling from the donor defect states. The total emission from the mixed monolayer is also enhanced with a relative quantum yield of $160 \%$ achieved. The results show a strong concentration dependence of the recycling effect, which can be optimised by tuning the donor:acceptor ratio. FRET in QD structures can be used to improve the efficiency of nanocrystal QD light emitting devices.

\section{ACKNOWLEDGEMENTS}

This work was financially supported by Science Foundation Ireland 10/IN.1/12975.

\section{REFERENCES}

[1] D.V. Talapin et al.: Prospects of colloidal nanocrystals for electronic and optoelectric applications, Chem. Rev. 1101389 (2011).

[2] S. V. Gapenko, Optical properties of semiconductor nanocrystals (Cambridge University Press, Cambridge, 1998).

[3] T. Förster: Zwischenmolekulare energiewanderung und fluoreszenz, Annalen Der Physik 2, $55-57$ (1948).

[4] A. A. Mamedov et al.: Nanorainbows: graded semiconductor films from quantum dots, J. Am. Chem. Soc. 123, 7783 (2001).

[5] N. Cicek et al.: Structural tuning of color chromaticity through nonradiative energy transfer by interspacing CdTe nanocrystal monolayers, Appl. Phys. Lett. 94, 061105 (2009).

[6] A. A. Chistyakov et al.: Interaction of $\mathrm{CdSe} / \mathrm{ZnS}$ core-shell semiconductor nanocrystals in solid thin films, Laser Phys. 16, 1625 (2006).

[7] T. Franzl, et al.: Exciton recycling in graded gap nanocrystal structures, J. Nano Lett. 4, 1599-1603 (2004).

[8] S. Nizamoglu et al.: Quantum efficiency enhancement in nanocrystals using nonradiative energy transfer with optimised donor-acceptor ratio for hybrid LEDs, Appl. Phys. Lett. 94, 243107 (2009).

[9] S. J. Byrne, et al.: Optimisation of the synthesis and modification of CdTe quantum dots for enhanced live cell imaging, J. Mater. Chem. 16, 2896-2902 (2006); A. L. Rogach, et al.,: Aqueous synthesis of thiolcapped CdTe nanocrystals: State-of-the-art, J. Phys. Chem. C 111, 14628-14637 (2007).

[10] G. Decher: Fuzzy Nanoassemblies: toward layered polymeric multicomposites, Science 227, 1232-1237 (1997).

[11] Lunz et al.: Two-dimensional Förster resonant energy transfer in mixed quantum dot monolayers: experiment and Theory, J. Phys. Chem. C 113, 3084-3088 (2009).

[12] W.W. Yu et al: Formation and stability of size-, shape-, and structure-controlled CdTe nanocrystals: ligand effects on monomers and nanocrystals, Chem. Mater. 15, 2845 (2003).

[13] M. Lunz et al.: Influence of quantum dot concentration on Förster resonant energy transfer in monodispersed nanocrystal quantum dot monolayers, Phys. Rev. B 81, 205316 (2010).

[14] M. Lunz, et al.: Donor Concentration Dependence of FRET Efficiency in Nanocrystal Quantum Dot Structures, Phys. Rev. B 83, 115423 - 1-10 (2011).

[15] B. W. Van der Meer, G. Coker and S. Y. S. Chen, Resonance energy transfer: theory and data (VCH, New York; Cambridge, 1994). 